\title{
Cadernode Pesquisa
}

Https://online.unisc.br/seer/index.php/cadpesquisa

ISSN on-line: $1677-5600$

Doi: 10.17058/cp.v30i1.12195

Universidade de Santa cruz do Sul - Unisc

Autor para contato: lobo@unisc.br

\section{Avaliação da qualidade da água na Bacia Hidrográfica do Arroio Preto, Santa Cruz do Sul, RS, Brasil}

Water quality evaluation in the Preto Stream Hydrographic Basin, Santa Cruz do Sul, RS, Brazil

\author{
Patrik Gustavo Wiesel \\ Elias Dresch
}

Universidade de Santa Cruz do Sul - UNISC - Santa Cruz do Sul - Rio Grande do Sul - Brasil

\author{
Eduardo Rodrigo Ramos de Santana
}

Fundação Estadual de Proteção Ambiental- FEPAM - Santa Cruz do Sul - Rio Grande do Sul - Brasil

\author{
Eduardo Alexis Lobo
}

Universidade de Santa Cruz do Sul - UNISC - Santa Cruz do Sul - Rio Grande do Sul - Brasil

\section{Resumo}

A pesquisa objetivou avaliar a qualidade da água da Bacia Urbana do Arroio Preto, Santa Cruz do Sul, RS, aplicando o Índice de Qualidade da Água (IQA) adaptado para sistemas lóticos sul brasileiros, em três pontos de amostragem ao longo do Arroio, Ponto 1, trecho superior; Ponto 2, trecho intermediário; e Ponto 3 próximo à foz. Foram realizadas 19 campanhas entre 2007 e 2016, medindo: temperatura, $\mathrm{pH}$, turbidez, oxigênio dissolvido, demanda bioquímica de oxigênio, nitrato, nitrito, nitrogênio amoniacal total, fosfato, sólidos totais dissolvidos e coliformes termotolerantes. Os resultados indicaram que houve uma significativa diminuição da qualidade da água $(p<0,05)$, variando de uma nota IQA igual a $89,9 \pm 1,1$ no Ponto 1 , para $45,7 \pm 14,0$ no Ponto 2 , e $41,4 \pm 10,0$ no Ponto 3. O ponto de coleta 1 foi classificado como tendo um nível de IQA "bom", caracterizadas como águas de boa qualidade, enquanto que os pontos de coleta P2 e P3 foram classificados como tendo um nível de IQA "ruim", e foram considerados como pontos de coleta críticos com a pior qualidade da água. Concluímos que o tratamento de esgoto sanitário surge como a principal demanda da região em termos de políticas públicas ambientais.

\section{Abstract}

The research aimed at evaluating the water quality of the urban Hydrographical Basin of Preto Stream, county of Santa Cruz do Sul, RS, applying the Water Quality Index (WQI) adapted for southern Brazilian lotic systems, in three sampling sites along the stream, Point 1 , upper reaches, Point 2, intermediate reaches, and Point 3, lower reaches. 19 campaigns between 2007 and 2016 were realized, measuring: water temperature, $\mathrm{pH}$, turbidity, dissolved oxygen, biochemical oxygen demand, nitrate, ammonia nitrogen, phosphate, total solids dissolved and thermotolerant coliforms. The results indicated that there was a significant decreasing in water quality $(p<0.05)$, varying from a WQI score of $89,9 \pm 1,1$ in Point 1 , to $45,7 \pm 14,0$ in Point 2 , and $41,4 \pm 10,0$ in Point 3 . The Point 1 was classified as having a "good" WQI level, characterized as good quality waters, while sampling points 2 and 3 were classified as having a "poor" WQI level, and were considered as critical sampling points with the worst water quality. We conclude that sanitary sewage treatment is the main demand in the region in terms of environmental public policies.

\section{Palavras-chave}

Bacia urbana do Arroio Preto, RS. Esgoto doméstico. Índice da Qualidade da Água (IQA).

\section{Keywords}

Urban Hydrographical Basin of Preto Stream, RS. Domestic sewage. Water Quality Index

(WQI). 


\section{Introdução}

Devido a sua dimensão continental, o Brasil está dividido em cinco regiões delimitadas principalmente em função de suas características climáticas e fisiográficas: Norte, Nordeste, Centro-Oeste, Sudeste e Sul, cada qual apresentando particularidades no que se refere aos problemas relacionados à gestão dos recursos hídricos. Segundo Tundisi (2001), na região Sul há escassez de água apesar dos rios de grande porte e vazão, devido aos efeitos cumulativos dos processos ambientais e usos múltiplos que são de amplo espectro: agricultura, indústrias, piscicultura, navegação e recreação. Para agravar a situação, a pequena parcela de água doce disponível vem tendo sua qualidade degradada principalmente devido ao acelerado crescimento populacional observado nas últimas décadas (SPERLING, 1997).

As águas superficiais urbanas brasileiras invariavelmente recebem cargas de poluentes das mais diversas origens e o grau desta contaminação é indicador direto do impacto causado pela urbanização sobre estes recursos naturais. A sobrevivência humana está diretamente relacionada aos cursos d'água, ressaltando que a locação de cidades próximas aos rios ao longo da história são provas irrefutáveis. Se o uso da água é reconhecido como fundamental à sociedade, as águas servidas e cursos d'água integrantes da malha urbana da cidade não têm a mesma atenção (CALDERARI e MARAGNO, 2013).

Neste sentido, a caracterização da qualidade da água é uma das formas de se avaliar os impactos causados pela interferência humana em sistemas aquáticos, possibilitando seu manejo de forma mais adequada (CORADI et al., 2009). Para este propósito, o monitoramento da qualidade da água e a disponibilização de índices simplificados surgem na comunidade científica como uma alternativa de grande aceitação. Por exemplo, para a região sul do Brasil, Moretto et al. (2012) tomaram por base o Índice de Qualidade da Água (IQA), originalmente desenvolvido pela National Sanitation Foundation nos Estados Unidos e calibraram sua aplicação às condições ambientais que prevalecem na Bacia Hidrográfica do Rio Pardo, RS, destacando que a principal alteração se deu na importância dos pesos dos parâmetros utilizados no cálculo do índice.

Neste contexto, o objetivo da pesquisa foi avaliar a qualidade da água da Bacia Urbana do Arroio Preto, Santa Cruz do Sul, RS, Brasil, aplicando o IQA adaptado aos sistemas lóticos sul brasileiros por Moretto et al. (2012).

\section{Material e métodos}

O estudo foi realizado na Bacia Hidrográfica do Arroio Preto, inserida na cidade de Santa Cruz do Sul, localizada na porção central do estado do Rio Grande do Sul, distante aproximadamente $150 \mathrm{~km}$ da capital Porto Alegre, correspondente à zona urbana da cidade e que soma uma área aproximada de 1.434 ha (Fig. 1). As coordenadas geográficas desta bacia urbana no Datum Sirgas 2000 são: Norte: $29^{\circ} 41^{\prime} 8.70^{\prime \prime S} 52^{\circ} 24^{\prime} 39.42^{\prime \prime O}$; Leste: $29^{\circ} 42^{\prime} 31.80^{\prime \prime S}$ $52^{\circ} 23^{\prime} 41.84^{\prime \prime O}$; Sul: $29^{\circ} 43^{\prime} 41.55^{\prime \prime S} 52^{\circ} 25^{\prime} 52.22^{\prime \prime O}$; e Oeste: $29^{\circ} 42^{\prime} 44.51^{\prime \prime S} 52^{\circ} 26^{\prime} 40.86^{\prime \prime O}$. 


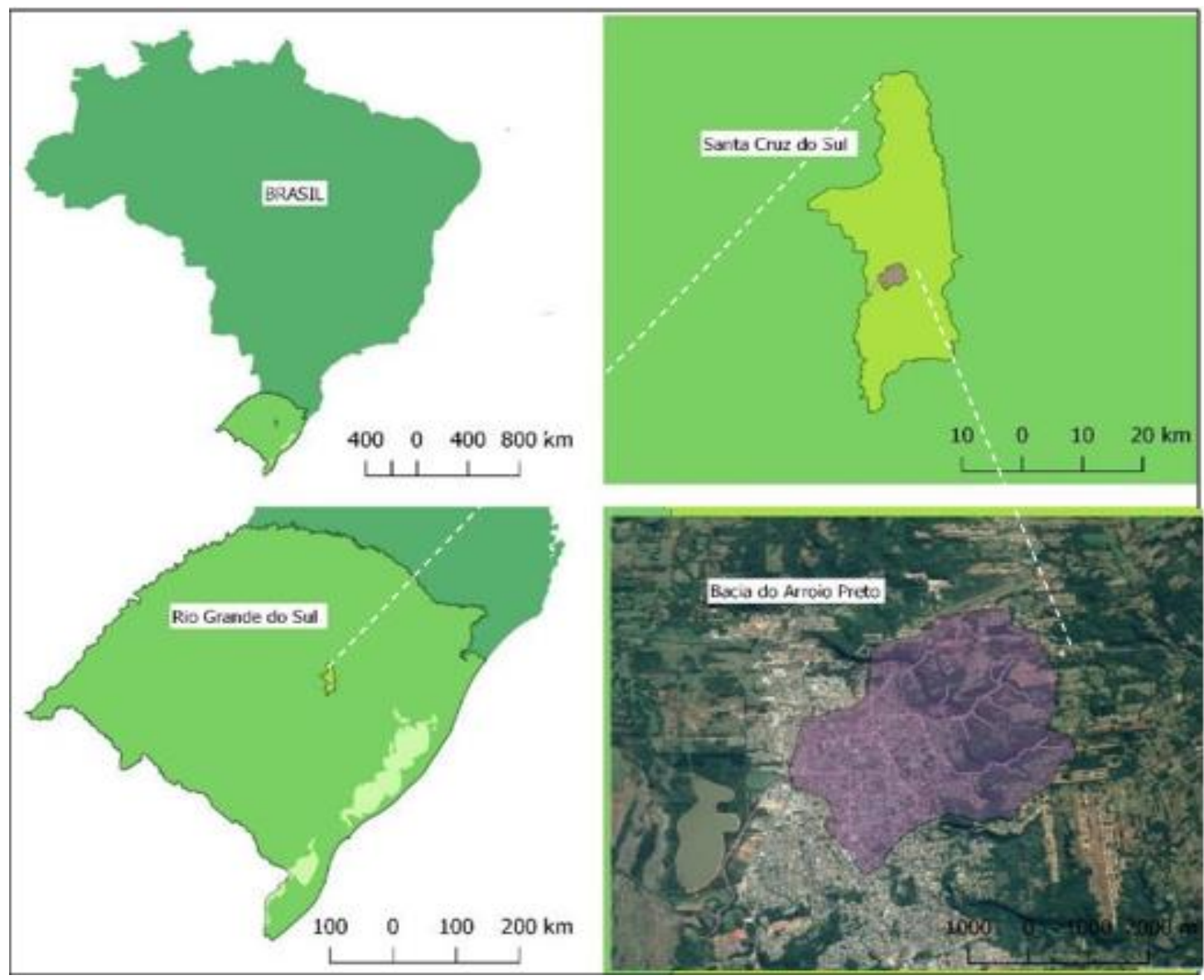

Figura 1. Localização do Municipio de Santa Cruz do Sul e da Bacia Hidrográfica do Arroio Preto no Estado do Rio Grande do Sul, Brasil.

As amostras para o estudo da qualidade da água foram localizadas em três pontos ao longo do leito principal do Arroio Preto (Fig. 2), que percorre entre suas zonas de nascentes, na porção leste da cidade, e seu desague no Rio Pardinho, cerca de 8,2 km. Ponto 1 (Coord. $29^{\circ} 42^{\prime} 38.22^{\prime \prime S} 52^{\circ} 24^{\prime} 24.98^{\prime \prime O}$ WGS84) no Parque da Gruta; Ponto 2 (29² $42^{\prime} 21.28^{\prime \prime S}$ $52^{\circ} 26^{\prime} 4.29^{\prime \prime O}$ WGS84) numa posição intermediária; e Ponto 3: $\left(29^{\circ} 42^{\prime} 44.73^{\prime \prime S} 52^{\circ} 26^{\prime} 40.67^{\prime \prime O}\right.$ WGS84) junto à BR471, próximo à foz.

Nos pontos de coleta selecionados foram realizadas 19 campanhas entre 2007 e 2016, sendo que os seguintes parâmetros de avaliação ambiental foram medidos: temperatura, $\mathrm{pH}$, turbidez, oxigênio dissolvido, demanda bioquímica de oxigênio após cinco dias, nitrato, nitrito, nitrogênio amoniacal total, fosfato, sólidos totais dissolvidos e coliformes termotolerantes. As técnicas utilizadas na coleta das amostras e na determinação analíticas das variáveis físicas, químicas e microbiológicas encontram-se descritas em APHA (2005).

Para o cálculo do Índice de Qualidade da Água (IQA), foi utilizado como base a pesquisa desenvolvida por Moretto et al. (2012), os quais calibraram o IQA originalmente desenvolvido pela National Sanitation Foundation dos Estados Unidos, adaptando-o às condições ambientais dos sistemas lóticos sul brasileiros, a partir da alteração dos pesos de importância das variáveis que compõem o índice (Tab. 1). A base de dados foi retirada dos estudos de monitoramento ambiental da Bacia do Preto desenvolvidos por Schuch et al. (2012) e pelo Laboratório de Limnologia da UNISC, entre 2007 a 2016, sendo que na ausência de algum dado das variáveis do IQA, houve uma redefinição percentual dos pesos das mesmas. Os cálculos do IQA foram feitos utilizando o Programa IQAData 2015 (POSSELT et al., 2015). A tabela 2 apresenta a interpretação do valor de IQA, que se classifica dentro de cinco faixas de qualidade da água, desde muito ruim até excelente. 


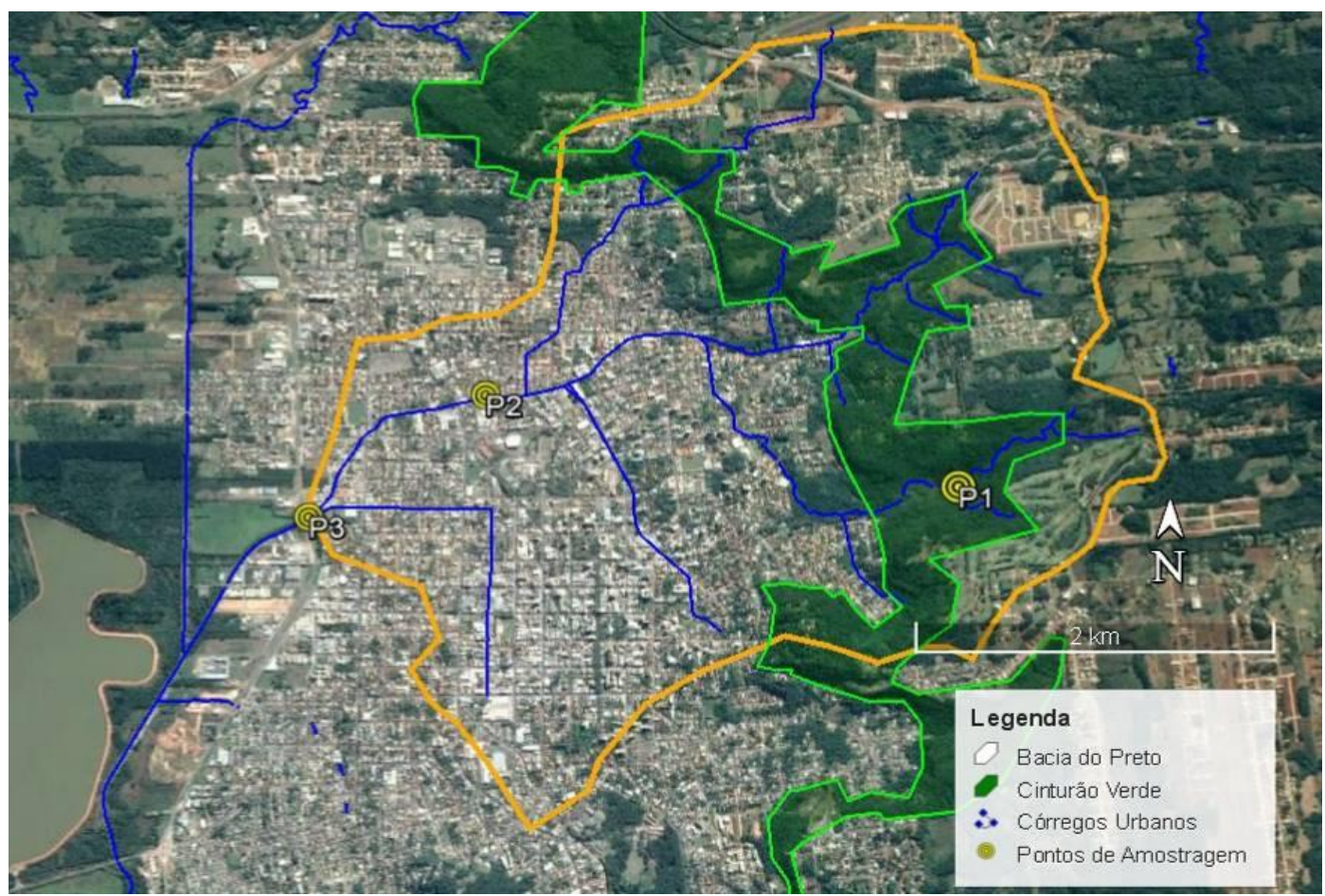

Figura 2. Mapa de localização dos pontos de coleta de amostras (P1, P2 e P3) para monitoramento da qualidade da água do Arroio Preto, RS, utilizando o Programa Google Earth PRO.

Tabela 1: Parâmetros e pesos relativos do IQA, conforme Moretto et al. (2012).

\begin{tabular}{|c|c|c|c|}
\hline Variáveis & Unidade & Peso NSF & Peso Calibrado \\
\hline Oxigênio dissolvido & \% de Saturação & 0,17 & 0,20 \\
\hline Coliformes Termotolerantes & NMP $100 \mathrm{~mL}^{-1}$ & 0,16 & 0,23 \\
\hline Potencial Hidrogeniônico - pH & - & 0,11 & 0,05 \\
\hline $\begin{array}{l}\text { Demanda Bioquímica de Oxigênio - } \\
\mathrm{DBO}_{5}\end{array}$ & $\mathrm{mg} \mathrm{L}^{-1}, \mathrm{O}_{2}$ & 0,11 & 0,08 \\
\hline Temperatura da água & ${ }^{\circ} \mathrm{C}$ & 0,10 & 0,03 \\
\hline Nitrato & $\mathrm{mg} \mathrm{L}^{-1}, \mathrm{NO}_{3}$ & 0,10 & 0,07 \\
\hline Fosfato & $\mathrm{mg} \mathrm{L}^{-1}, \mathrm{PO}_{4}$ & 0,10 & 0,16 \\
\hline Turbidez & uT & 0,08 & 0,12 \\
\hline Sólidos Dissolvidos Totais & $\mathrm{mg} \mathrm{L}^{-1}$ & 0,07 & 0,06 \\
\hline Somatório & & 1,0 & 1,0 \\
\hline
\end{tabular}

Tabela 2: Faixas de qualidade de água para o IQA.

\begin{tabular}{ll}
\hline Faixas de IQA & Classificação da qualidade da água \\
\hline $0-25$ & Muito ruim \\
$26-50$ & Ruim \\
$51-70$ & Regular \\
$71-90$ & Bom \\
$91-100$ & Excelente \\
\hline
\end{tabular}

\section{Resultados e discussões}

Os resultados obtidos (Fig. 3) indicaram que houve uma significativa diminuição da qualidade da água em termos do IQA ao longo do trajeto do Arroio Preto $(p<0,05)$, uma vez que a nota variou de $89,9 \pm 1,1(C V=1,2 \% ; n=4)$, no Ponto 1 , Parque da Gruta; para uma nota 
igual a $45,7 \pm 14,0(C V=30,6 \% ; n=5)$ no Ponto 2 , trecho intermediário, e uma nota igual a $41,4 \pm 10,0$ (CV = 25,5\%; $n=10)$.

O ponto de coleta 1, Parque da Gruta, foi classificado como tendo um IQA "bom", que corresponde a águas das Classes de Uso 1 e 2 da resolução 357/2005 do CONAMA (Conselho Nacional do Meio Ambiente) (CONAMA, 2005), caracterizadas como águas de boa qualidade (KLAMT et al., 2018), apropriadas para o consumo humano, após tratamento convencional, à proteção das comunidades aquáticas e à recreação de contato primário (balneabilidade), dentre as suas principais características (BRASIL, 2005).

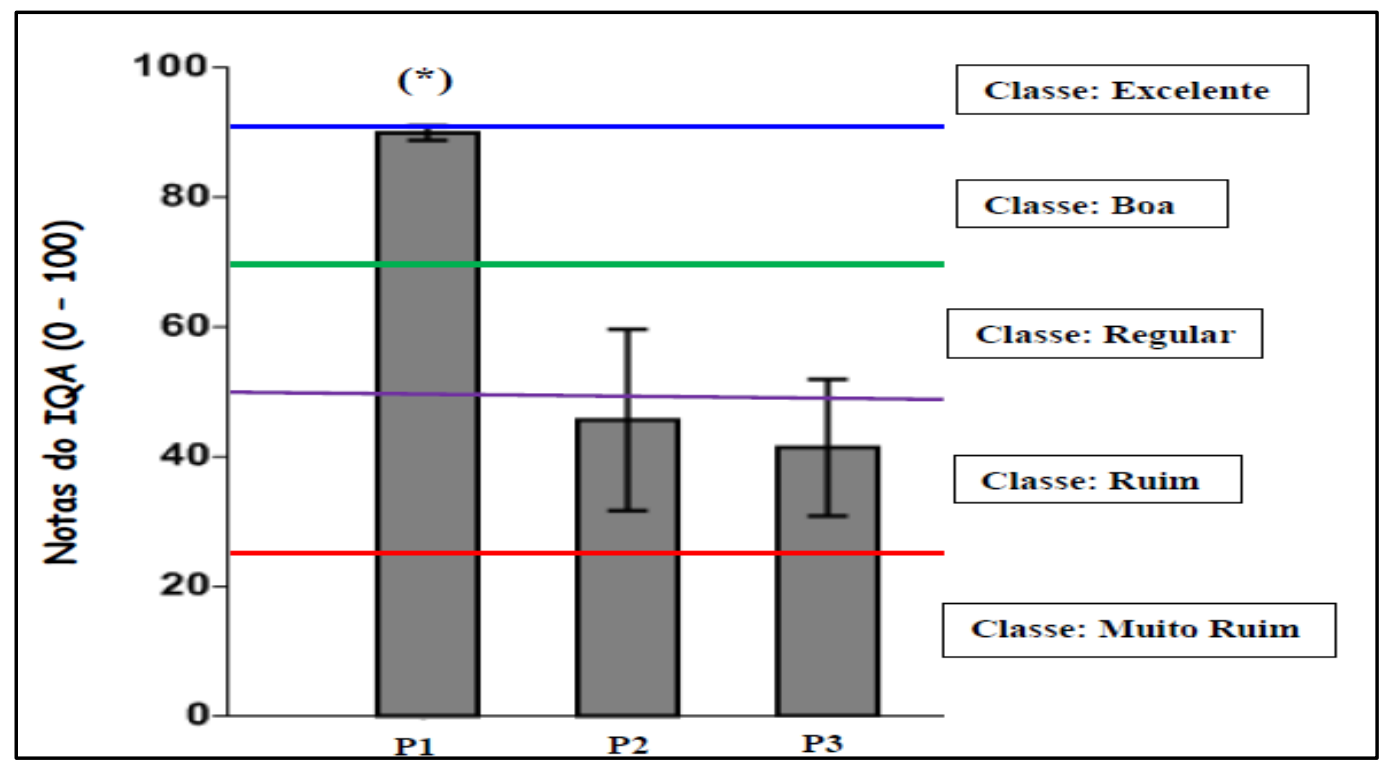

Figura 3. Média ( \pm desvio-padrão) do Índice e Qualidade da Água (IQA), adaptado por Moretto et al. (2012) para sistemas lóticos sul brasileiros, no ponto 1 (P1), Parque da Gruta, ponto 2 (P2), intermediário e ponto 3 (P3), foz, na Bacia do Arroio Preto, RS, em 19 campanhas realizadas entre 2007 e 2016. $\left({ }^{*}\right)$ : Diferença significativa $(p<0,05)$. Apresentam-se, também os limites para as classes de qualidade do IQA.

Já os pontos de coleta P2 e P3 foram classificados como tendo um IQA "ruim", que corresponde a águas da Classe de Uso 4 da resolução 357/2005 do CONAMA (CONAMA, 2005), considerados como pontos de coleta críticos por apresentarem a pior qualidade da água (KLAMT et al., 2018), destinada apenas à navegação e harmonia paisagística (BRASIL, 2005).

Estes resultados poderiam ser explicados considerando que estes pontos de coleta se encontram sujeitos ao impacto de uma série de atividades antrópicas locais, destacando principalmente a forte carga orgânica advinda de esgoto doméstico, bem como do excesso de fertilizantes e insumos agrícolas utilizados em lavouras. Estes impactos ambientais podem ser verificados a partir das variáveis que foram responsáveis pela classificação destes pontos de coleta no nível "ruim" (classe de uso do CONAMA 4), sendo elas o fosfato total (100,0\%, $n=12$ ) e coliformes termotolerantes $(83,3 \%, n=6)$. Desta forma, em função destas variáveis, concluise que a contaminação orgânica e a eutrofização da água são os principais problemas ambientais que caracterizaram este trecho do Arroio Preto.

Estes resultados vêm corroborar trabalhos de monitoramento ambiental em sistemas hídricos regionais realizados pela UNISC, os quais têm demonstrado que estes já apresentam estados bastante avançados de eutrofização (BES et. al., 2012; BÖHM et. al., 2013; HEINRICH et al., 2014; LOBO et. al., 2010, 2014, 2015, 2016a,b; SALOMONI et. al., 2011; SCHUCH et. al., 2012, 2015). Ainda, segundo Tundisi (2006), esta condição caracteriza de forma generalizada 
os cursos d'água em toda a região Sul do Brasil, conforme resultados obtidos pelo Projeto Brasil das Águas.

Todavia, os resultados do IQA no Ponto $3(41,4 \pm 10,0-C V=25,5 \%, n=10)$, correspondente ao trecho próximo a foz da Arroio Preto no Rio Pardinho, indicam que corresponde ao ponto mais crítico do ecossistema, uma vez que concentra toda a carga poluente acumulada ao longo do seu percurso, antes de entrar no seu corpo receptor, e serve de alerta, indicando um diagnóstico de sobrecarga de poluentes nos córregos urbanos da Bacia. A urbanização e outras atividades humanas, sem planejamento, acabam prejudicando, muitas vezes de forma irreversível, a resiliência de um curso de água. De fato, a baixa variabilidade do valor do IQA observado no ponto 3, 25,5\% na série temporal 2007/2016 ( $n=$ 10), indica uma tendência de constância neste cenário, caracterizando uma fonte de poluição continua.

Concluímos que o alto grau de degradação da qualidade das águas superficiais observado ao longo da Bacia do Preto, sinaliza claramente que o tratamento de esgoto sanitário e seus impactos surgem como a principal demanda atual da região, e deve ser considerada uma prioridade em termos de politícas públicas ambientais.

\section{Agradecimentos}

Os autores agradecem ao Conselho Nacional de Desenvolvimento Científico e Tecnológico (CNPq) pela concessão de Bolsa de Iniciação Científica PIBIC/CNPq ao primeiro autor deste trabalho.

\section{Referências}

1. APHA. American Public Health Association. Standard Methods for the Examination of water and Wastewater. 21 ed. Washington. 2005.

2. BES, D.; LUC, E.; TORGAN, L.C.; LOBO, E.A. Composition of the epilithic diatom flora from a subtropical river, Southern Brazil. Iheringia Série Botânica, v. 67, n. 1, p. 93-125, 2012.

3. BÖHM, J. S.; SCHUCH, M.; DÜPONT, A.; LOBO, E.A. Response of Epilithic Diatom Communities to Downstream Nutrient Increases in Castelhano Stream, Venâncio Aires City, RS, Brazil. Journal of Environmental Protection, v. 4, p. 20-26, 2013.

4. BRANCO, S. M. Água: origem, uso e preservação. 13. ed. São Paulo: Editora Moderna. 2005.

5. BRASIL. Conselho Nacional do Meio Ambiente. Resolução n. 357, de 17 de março de 2005. Diário Oficial da República Federativa do Brasil, Brasília, DF, 18 de março de 2005. Disponível em: http://www.mma.gov.br/port/conama/res/res05/res35705.pdf.

6. CALDERARI, E.S.; MARAGNO, A.F. Águas urbanas: um novo olhar para o planejamento urbano nas cidades. Revista Nacional de Gerenciamento de Cidades, v. 1, n. 2, p. 20-41, 2013.

7. CORADI, P.C.; FIA, R.; PEREIRA-RAMIREZ, O. Avaliação da qualidade da água superficial dos cursos de água do município de Pelotas, RS, Brasil. Revista Ambiente \& Água, v. 4, n. 2, p. 46-56, 2009.

8. HEINRICH, C.G.; LEAL, V.L.; SCHUCH, M.; DÜPONT, A.; LOBO, E.A. Epilithic diatoms in headwater areas of the hydrographical sub-basin of the Andreas Stream, RS, Brazil, and 
their relation with eutrophication processes. Acta Limnologica Brasiliensia, v. 26, n. 4, p. 347-355. 2014.

9. KLAMT, R.A.; DELEVATI, D.M.; COSTA, A.B.; LOBO, E.A. Evaluation of water resource preservation areas in the Hydrographical Basin of Andreas Stream, RS, Brazil, using environmental monitoring programs. Water International (In press). 2018.

10. LOBO, E.A.; WETZEL, C.E.; SCHUCH, M.; ECTOR, L. Diatomáceas epilíticas como indicadores da qualidade da água em sistemas lóticos subtropicais e temperados brasileiros. Santa Cruz do Sul: EDUNISC, 2014.

11. LOBO, E.A.; SCHUCH, M.; HEINRICH, C.G.; COSTA, A.B.; DÜPONT, A.; WETZEL, C.E.; ECTOR, L. Development of the Trophic Water Quality Index (TWQI) for subtropical temperate Brazilian lotic systems. Environmental Monitoring Assessment, v. 6, p. 1-13, 2015.

12. LOBO, E.A.; HEINRICH, C.D.; SCHUCH, M.; WETZEL, C.E.; ECTOR, L. Diatoms as bioindicators in rivers. p. 245-271. In: NECCHI Jr. (Ed.). River Algae. Springer International Publishing. 2016a.

13. LOBO, E.A.; HEINRICH, C.D.; SCHUCH, M.; DÜPONT, A.; COSTA, A.B.; WETZEL, C.E.; ECTOR, L. Índice trófico da qualidade da água: Guia ilustrado para sistemas lóticos subtropicais e temperados brasileiros. Santa Cruz do Sul: EDUNISC. E-Book (www.unisc.br/edunisc). 2016b.

14. MORETTO, D.L.; PANTA, E.; COSTA, A.B.; LOBO, E.A. Calibration of Water Quality Index (WQI) based on Resolution no 357/2005 of the Environment National Council (CONAMA) Brazil. Acta Limnologica Brasiliensia, v. 24, n. 1, p. 29-42, 2012.

15. POSSELT, E. L.; COSTA, A.B.; LOBO, E.A. Software IQAData 2015. Registro no INPI BR 512015000890-0. Programa de Mestrado em Sistemas e Processos Industriais (PPGSPI), Programa em Tecnologia Ambiental - Mestrado e Doutorado (PPGTA), UNISC. Disponível em: http://www.unisc.br/ppgspi. 2015.

16. SCHUCH, M.; ABREU-JUNIOR, E.; LOBO, E.A. Water quality evaluation of urban streams in Santa Cruz do Sul City, RS, Brazil. Bioikos, Campinas, v. 26, n. 1, p. 3-12, 2012.

17. SCHUCH, M.; OLIVEIRA, M.A.; LOBO, E.A. Spatial Response of Epilithic Diatom Communities to Downstream Nutrient Increases. Water Environment Research, v. 87, n. 6, p. 547-558, 2015.

18. SPERLING, M. Princípios do Tratamento Biológico de Águas Residuárias - Lodos Ativados. Minas Gerais: ABES, 1997.

19. TUNDISI, J. G. Gerenciamento da qualidade da água: interações entre pesquisa, desenvolvimento tecnológico e políticas públicas. Revista Brasileira de Pesquisa e Desenvolvimento, v. 3. n. 2, p. 57-68, 2001.

20. TUNDISI, J. G. O Futuro dos Recursos Hídricos no Brasil. Projeto Brasil das Águas. Disponível em http://www.brasildasaguas.com.br/. 2006. 